\title{
A comparative analysis of machine learning methods for classification type decision problems in healthcare
}

\author{
Nahit Emanet ${ }^{1}$, Halil R Öz ${ }^{2}$, Nazan Bayram ${ }^{3}$ and Dursun Delen ${ }^{4^{*}}$
}

\author{
* Correspondence: dursun.delen@ \\ okstate.edu \\ ${ }^{4}$ Department of Management \\ Science and Information Systems, \\ Spears School of Business, \\ Oklahoma State University, \\ Stillwater, OK, USA \\ Full list of author information is \\ available at the end of the article
}

\begin{abstract}
Advanced analytical techniques are gaining popularity in addressing complex classification type decision problems in many fields including healthcare and medicine. In this exemplary study, using digitized signal data, we developed predictive models employing three machine learning methods to diagnose an asthma patient based solely on the sounds acquired from the chest of the patient in a clinical laboratory. Although, the performances varied slightly, ensemble models (i.e., Random Forest and AdaBoost combined with Random Forest) achieved about $90 \%$ accuracy on predicting asthma patients, compared to artificial neural networks models that achieved about $80 \%$ predictive accuracy. Our results show that noninvasive, computerized lung sound analysis that rely on low-cost microphones and an embedded real-time microprocessor system would help physicians to make faster and better diagnostic decisions, especially in situations where x-ray and CT-scans are not reachable or not available. This study is a testament to the improving capabilities of analytic techniques in support of better decision making, especially in situations constraint by limited resources.
\end{abstract}

Keywords: Classification; Data mining; Machine learning; Decision making; Asthma; Pulmonary sound signals; Discrete wavelet transformation

\section{Background}

As the decision situations become increasingly more complex, advanced analytical techniques are gaining popularity in addressing wide variety of problem types (descriptive, predictive and prescriptive) in many fields including healthcare and medicine (Delen et al. 2009). Because of the rapid increase in the collection and storage of large quantities of data (facilitated by improving software and hardware capabilities coupled with increasingly lower cost of acquiring and using them), data and model driven decision making (a.k.a. analytics) is becoming a mainstream practice in every field imaginable (from art to business, medicine to science). One area where faster and better decisions could make a significant difference is in healthcare/medicine. This data rich field can undoubtedly use what modern day decision analytics has to offer (Oztekin et al. 2009). In this study, we used analytics to address a classification type decision problem, namely prediction of asthma using only the chest sound signals obtained from actual patients using ordinary microphones.

\section{Springer}

(c) 2014 Emanet et al.; licensee Springer. This is an open access article distributed under the terms of the Creative Commons Attribution License (http://creativecommons.org/licenses/by/2.0), which permits unrestricted use, distribution, and reproduction in any medium, provided the original work is properly cited. 
Auscultation of pulmonary sounds provides invaluable clinical information on the health of the respiratory system. It is known in medicine that sounds emanating from the respiratory system are correlated with the underlying pulmonary pathology. The changes in lung structure change the spectrum of sounds heard over the chest wall. In addition to the typical sounds associated with the breathing process, extra or additional sounds are heard over the normal pulmonary sounds. These additional sounds are called adventitious pulmonary sounds and detection of these adventitious sounds is an important part of the respiratory examination that allows the physician to detect some pathological diseases.

Adventitious pulmonary sounds can be divided into five categories: wheezes, crackles, stridors, squawks and rhonchi. Although stethoscope is widely used by physicians as a simple, non-invasive tool for the auscultation of pulmonary sounds, it has been regarded as a tool with low diagnostic value, not only because pulmonary sounds for each patient are significantly different and they change for the same patient over time, but also because it is a subjective process that depends on the experience and hearing capability of physician. Stethoscope is not an ideal acoustic instrument either; it attenuates frequency components of pulmonary sounds above $120 \mathrm{~Hz}$ by making it impossible for the physician to hear pathological sounds of higher frequencies. Moreover, auscultation with stethoscope does not allow long term monitoring of pulmonary sounds.

Electronic auscultation of pulmonary sounds (Earis and Cheetham 2000), on the other hand, is a reliable and quantitative method that eliminates the shortcomings of stethoscope. In this system, a microphone placed at designated locations on the chest of a patient provides non-stationary pulmonary sound signals which can be recorded for an extensive period of time for subsequent analysis. Significant diagnostic information can be obtained from the frequency distribution of these signals.

The ultimate goal of this work is to develop a fully automated, highly accurate, lowcost and easy to use diagnostic tool for pulmonary diseases as a decision support tool for a physician. However, in this paper, we initially restrict our efforts on studying and diagnosing asthma, because of the prevalence of asthma in the world as one of the highest among all pulmonary disorders. It is estimated that 300 Million people worldwide suffers from asthma (Masoli et al. 2010) and by 2025, the number of patients expected to exceed 100 Million. The symptoms of asthma include wheezing, shortness of breath, chest tightness and cough. Wheezes are characterized by periodic waveforms with a dominant frequency greater than $100 \mathrm{~Hz}$, and lasting for longer than $150 \mathrm{~ms}$ (Oz et al. 2010). Multiple monophonic wheezes occurring simultaneously in a patient is known as a classic symptom for asthma disease (Ali et al. 2009).

In the literature, a large number of studies have focused on classifying pulmonary diseases. Doyle (1994) tried to classify adventitious pulmonary sounds (crackles, wheezes, pleural-friction rub and stridor) using artificial neural network with a reported accuracy of $83 \%$. Sankur et al. (1994) build classification models to differentiate between normal and adventitious pulmonary sounds using auto-regression models, and achieved a prediction accuracy of $87 \%$. Gavriely (1995) tested and demonstrated the effectiveness of computerized pulmonary sound analysis in addition to existing spirometry pulmonary function test. Pesu et al. (1998) used a wavelet packet-based method for the detection of adventitious pulmonary sounds, and learning vector quantization (LVQ) for the classification with limited success on classification accuracy. Kandaswamy et al. (2004) 
applied wavelet transform for the time-frequency analysis of pulmonary sound signals; used variety of artificial neural networks architectures for the classification; and achieved classification accuracy as high as $94 \%$. Murphy et al. (2004) focused on pneumonia and included a customized pneumonia score to features obtained from sound analysis, and time expanded waveform analysis is employed for automated classification. Similarly, after two years later, Kahya et al. (2006) also included customized parameters such as crackles parameters based on the duration of crackles during a breath cycle, and employed $k$-nearest neighbor algorithm for automatic classification, and archiving acceptable prediction accuracy. Ono et al. (2009) focused on interstitial pneumonia (IP) and investigated inspiratory lung sounds with Fast Fourier transformation to convert the data into a machine usable digitized format. Mohammed (2009), in order to classify pulmonary sounds, used cepstral analysis and Gaussian mixture models and achieved prediction accuracy close to 90\%. Oz et al. (2009) combined Fast Fourier transformation with genetics programming (GP) and Fuzzy C-Means clustering to analyze pulmonary diseases. They also achieved prediction accuracy close to $90 \%$.

Many of the above mentioned studies employed feature extraction techniques based on wavelet transformation, Fast Fourier transformation, Mel-frequency cepstral coefficients. For the automatic classification they employed artificial neural networks, Gaussian mixture models, learning vector quantization, genetics programming, k-nearest neighbor, among others.

In this study, we used a four-stage process to analyzed pulmonary sound signals: normalization, wavelet decomposition, feature extraction, and the classification (where we classified respiratory sounds using Random Forest algorithm, AdaBoost combined with Random Forest and artificial neural networks). Pulmonary sounds recorded from various subjects are normalized so that they would have approximately the same loudness level irrespective of the subject and/or environmental conditions. After normalization, feature vectors are formed by using this normalized data. The signals are decomposed into frequency sub-bands using discrete wavelet transformation (DWT) (Mallat 2009, Jensen and Harbo 2001 and Daubechies 1990. A set of statistical features is extracted from these sub-bands to represent the distribution of wavelet coefficients. These statistical feature vectors then introduced to the classification algorithms to evaluate the pulmonary sound signals as either normal or asthma. Block diagram of the diagnostic system is depicted in Figure 1.

\section{Methods}

\section{System description and data collection}

All pulmonary sounds were recorded at University of Gaziantep, Faculty of Medicine. In particular, recording of respiratory sounds was conducted in a clinical laboratory. Respiratory acoustic signals were recorded using Sony ECM-T150 electret condenser microphones with air coupler applied over right and left posterior bases of the lungs: positions $\mathrm{P} 4$ and $\mathrm{P} 5$, respectively. These air coupled microphones had linear frequency response between $50 \mathrm{~Hz}$ and $15 \mathrm{KHz}$. They were attached to the body of the subjects at the aforementioned positions.

Amplification and band pass filtering (Kester 2005) was performed prior to analogueto-digital conversion with a 16-bit resolution at a sampling rate of $8 \mathrm{~K}$ samples/s per channel in order to remove environmental sounds, heart and muscle sounds, and 


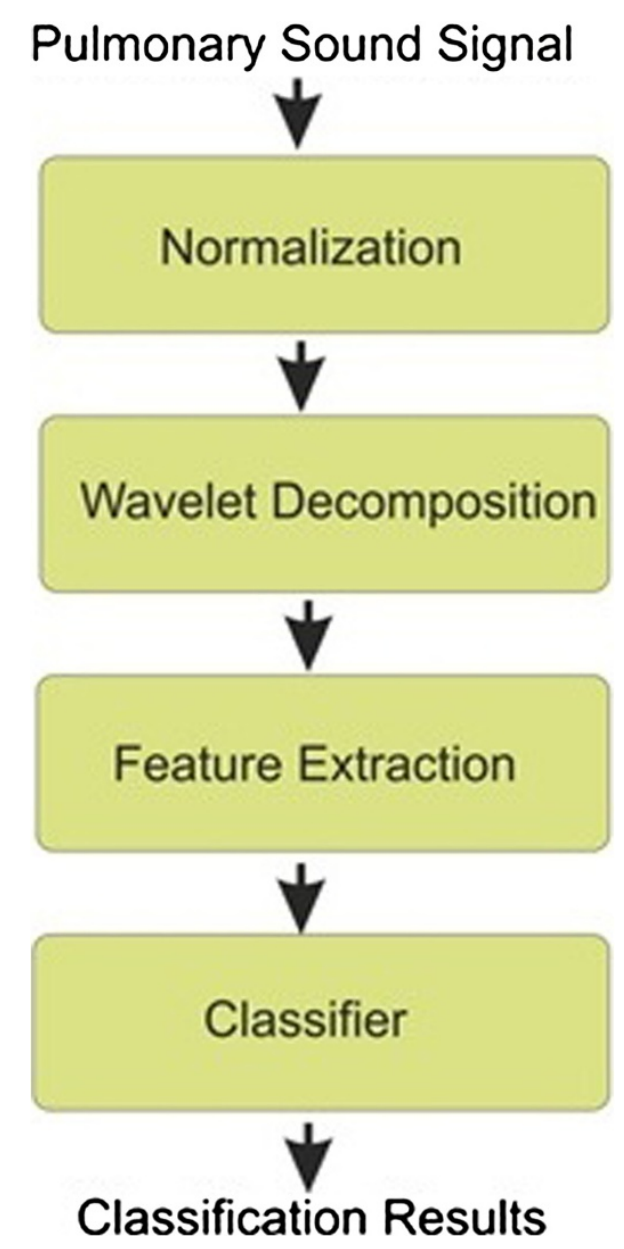

Figure $1 \mathrm{~A}$ high-level conceptual diagram of the diagnostic process.

friction sounds caused by the movements of microphones. SSM2019, OP275 and MAX295 integrated circuits are used in the amplification and band pass filtering circuit as shown in Figure 2. SSM2019 is a very low noise microphone preamplifier, which is often used to amplify microphone's low output voltage, typically in the 0 to 100 microvolt range, to a level that is usable for recording. The gain of the preamplifier was set to 11 by $1 \mathrm{~K} \Omega$ metal film resistor to obtain minimum total harmonic distortion. The filtering stage comprises both an active high pass Bessel filter of order six and an active low pass Butterworth filter of order eight. While Butterworth filter was optimized for maximal gain flatness, Bessel filters provided low overshoot and fast settling. High pass filter with cut-off frequency of $100 \mathrm{~Hz}$ was realized by three Analog Devices OP275 operational amplifiers. High pass filter was followed by a low pass filter with cut-off frequency of $2 \mathrm{KHz}$. It was implemented by using MAX295 8th-Order SwitchedCapacitor Filter.

After pulmonary sound signals are acquired from right and left posterior bases of the lungs, they are digitized and digitally filtered for further noise removal. Since inspiration and expiration phases have different information, the signals are divided into those parts separately. 


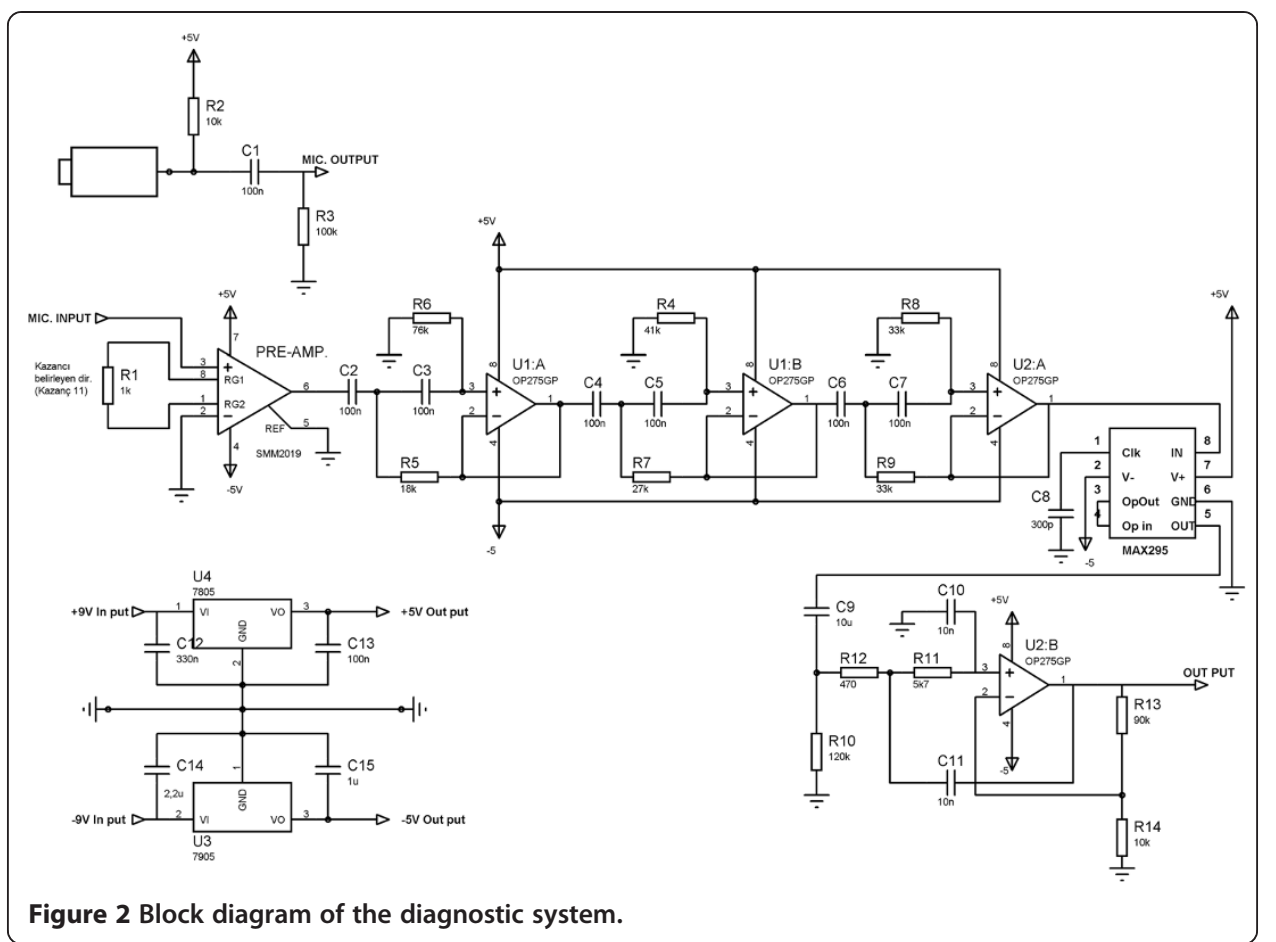

Ten healthy and ten asthmatic patients were studied during the data collection phase. The specifics about those twenty subjects are shown in Table 1. All recordings were performed under the supervision of a senior physician who was specialized in pulmonary diseases using an ARM based mobile biomedical data acquisition device (Fuber 2000 and Catmakas et al. 2009). The number of sound recordings from the 20 subjects was 40 in total. The ages of the subjects were in the range of 16 to 62 . There were 6 smokers and 14 nonsmokers. Seven subjects were male and 13 were female.

Though listed in Table 1, in the formulation of this study, only the sound signals are used as independent variables; none other special condition (e.g., socio-demographic characteristics) like age, sex, weight, and smoking habits were taken into consideration.

Percentage of forced vital capacity (FVC) and forced expiratory volume in one second (FEV1) values, which are used in pulmonary function test (PFT), are also shown in Table 1. During clinical evaluation, subjects were asked to inhale as deeply as possible up to their total lung capacity and then exhale completely as fast as and as hard as possible. The volume change of the lung during exhalation is called as FVC. The FEV1 is also defined as the volume exhaled during the first second of FVC.

Asthma is a common disease characterized by inflammation and hyper-reactivity in the airways which causes reversible airflow limitation. Patients with well controlled asthma demonstrate normal breath sounds and lung function whereas in poorly controlled asthma or during exacerbations, wheezing is usually heard in parallel with a fall in FEV1 and FEV1/FVC.

The ratio of FEV1 to FVC is critical in defining lung diseases characterized by airflow limitation such as asthma, chronic obstructive lung disease, obliterative bronchiolitis, 
Table 1 Clinical values about the subjects

\begin{tabular}{|c|c|c|c|c|c|}
\hline Age & Gender & Smoke & FEV1\% & FVC\% & Diagnosis \\
\hline 49 & $\mathrm{~F}$ & No & 38 & 84 & Asthma \\
\hline 62 & $F$ & Yes & 108 & 113 & Asthma \\
\hline 48 & M & Yes & 32 & 79 & Asthma \\
\hline 60 & $F$ & No & - & - & Asthma \\
\hline 59 & $F$ & No & 39 & 69 & Asthma \\
\hline 55 & $F$ & No & 68 & 89 & Asthma \\
\hline 55 & F & No & 37 & 50 & Asthma \\
\hline 34 & $\mathrm{~F}$ & No & 70 & 93 & Asthma \\
\hline 55 & $F$ & No & 132 & 125 & Asthma \\
\hline 16 & M & No & 99 & 106 & Asthma \\
\hline 48 & $F$ & No & - & - & Normal \\
\hline 41 & M & No & 96 & 98 & Normal \\
\hline 18 & $\mathrm{~F}$ & No & 105 & 102 & Normal \\
\hline 40 & M & No & 90 & 101 & Normal \\
\hline 29 & $\mathrm{~F}$ & Yes & 88 & 106 & Normal \\
\hline 24 & M & No & 107 & 110 & Normal \\
\hline 35 & M & Yes & 106 & 118 & Normal \\
\hline 30 & $F$ & Yes & 101 & 111 & Normal \\
\hline 30 & $\mathrm{~F}$ & Yes & 111 & 125 & Normal \\
\hline 37 & M & No & 110 & 144 & Normal \\
\hline
\end{tabular}

and cystic fibrosis. In general, FEV1/FVC ratio less than $70 \%$ is indicative of airflow limitation but this level is higher for younger individuals and decreases with age. In the instances where lung volumes are diminished but the FEV1/FVC ratio is preserved, restrictive lung pathology such as interstitial lung disease, pleural effusion or chest wall deformities may be suspected.

Figures 3, 4, 5, 6 represent pulmonary sound signal waveforms recorded from both healthy and asthmatic subjects during the inspiration and expiration cycles.

\section{Feature extraction}

A critical phase in any prediction model development effort is the characterization and transformation of the original data (sound signals, in this case) into a form that is most appropriate to the machine learning models being used. In this study, original pulmonary sound signal vectors were formed by discrete sample points. Mathematical transformations are applied to these signals in order to obtain further information that is not readily available in the original raw signal. Some of the most widely used transformation methods include linear transformations, such as principle component analysis (PCA) and linear discriminant analysis (LDA) (Martinez and Kak 2001 and Breiman 2001). Although PCA and LDA were very commonly used in sound signal transformation operations, they are not necessarily the best ones. In fact, for non-stationary signals, a wavelet-based time-frequency representation may be used for feature extraction. The basic idea of the discrete wavelet transform is to represent any arbitrary function $f$ as a superposition of wavelets. Any such superposition decomposes $f$ into different 


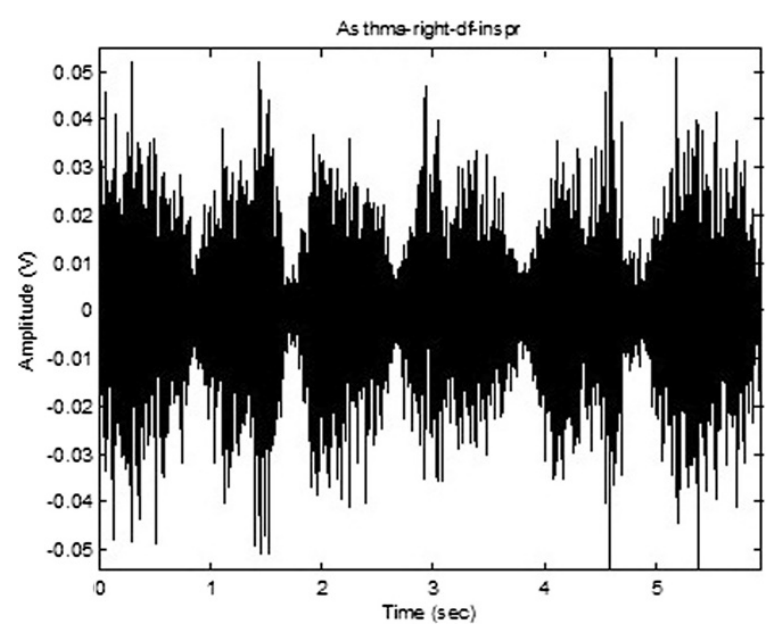

Figure 3 Inspiration cycle after digital filtering for an asthmatic patient.

scale levels, where each level is then further decomposed with a resolution adapted to the level.

The discrete wavelet transformation of a signal, $f[\mathrm{n}]$, is calculated by passing it through two digital filters and two down-samplers by 2. Digital filters are low-pass filter, $h$, and its complementary high-pass filter, $g$. The down-sampled outputs of high-pass and low-pass filters provide the detail signal, D, and the approximation signal, A, respectively as depicted in Figure 7. While this transform reduces the time resolution of the output signal by half, it doubles its frequency resolution.

This decomposition was recursively repeated to further increase the frequency resolution. At each level, approximation coefficients was decomposed with high and low pass filters and then down-sampled. The procedure of 3-level decomposition of a signal $f[\mathrm{n}]$ is represented as a binary tree as depicted in Figure 8. In order to perform wavelet

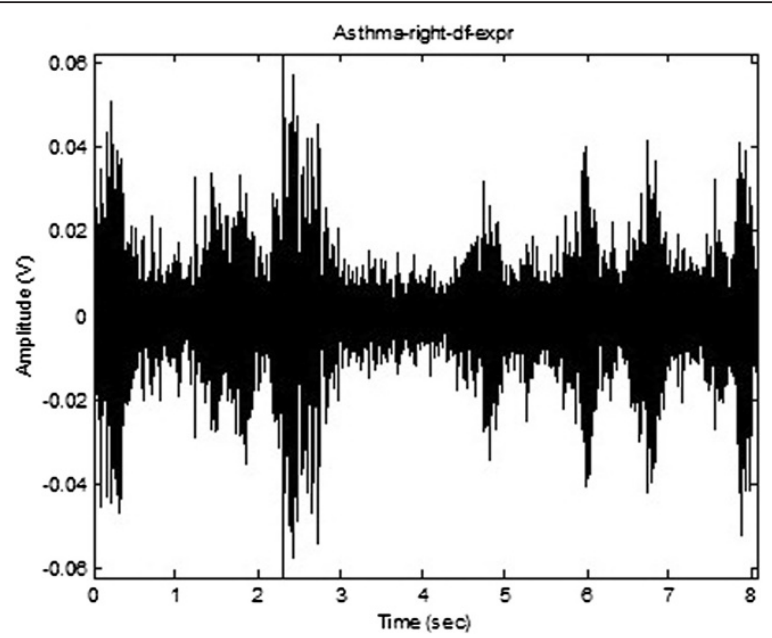

Figure 4 Expiration cycle after digital filtering for an asthmatic subject. 


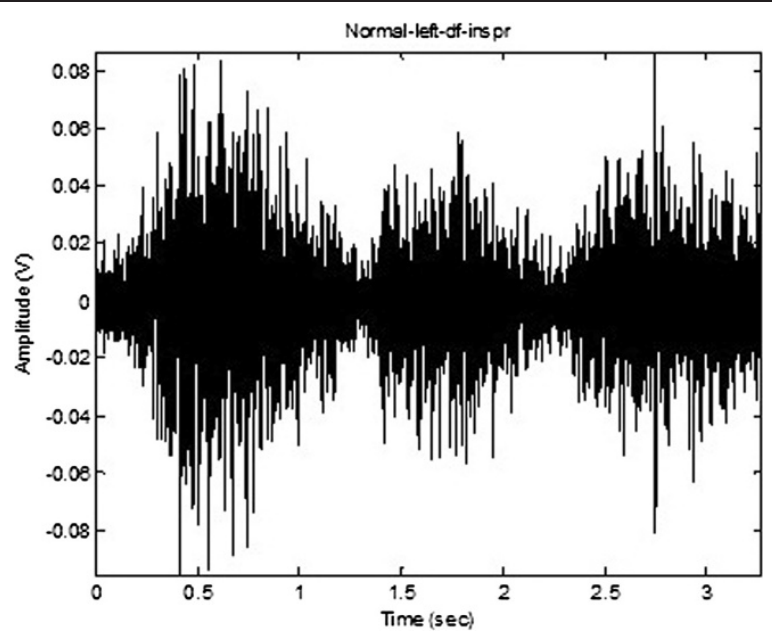

Figure 5 Inspiration cycle after digital filtering for a healthy subject.

analysis of pulmonary sounds, Matlab program was used. Daubechies db8 with 6 levels (Daubechies 1990) was used as the mother wavelet in the analysis.

Selection of the number decomposition levels is very important in the analysis of signals using DWT. The levels were chosen in such a way that frequency sub-bands of the decomposed signal correlated well with the frequencies required for the classification. Since the pulmonary sounds do not have any useful frequency components below 60 $\mathrm{Hz}$ and above $2000 \mathrm{~Hz}$, the number of levels was chosen as six by taking into consideration the sampling frequency of $8 \mathrm{KHz}$. Therefore, the signal was decomposed into the details D1-D6 and one final approximation A6. Figure 9 depicts the pulmonary signal, $s$, recorded from the right posterior base of a healthy subject. The decomposition is done by using Daubechies db8 wavelet with 6 levels. The ranges of frequency subbands are given in Table 2.

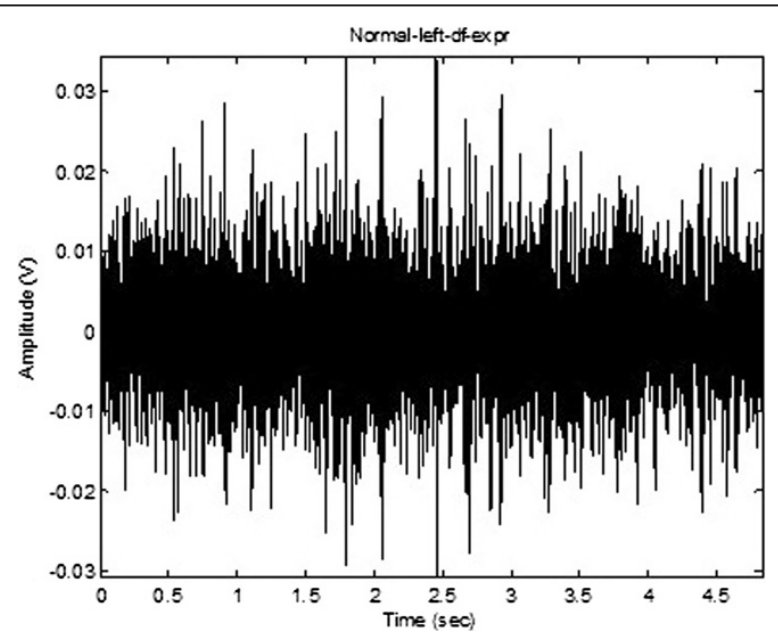

Figure 6 Expiration cycle after digital filtering for a healthy subject. 


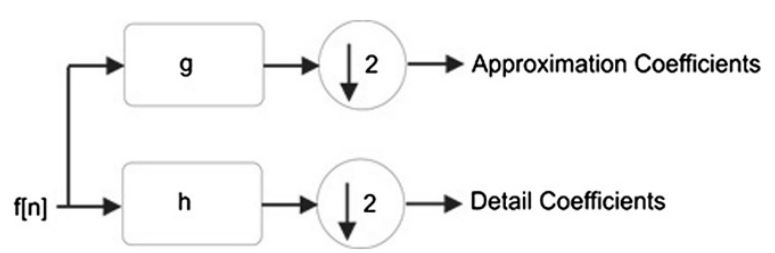

Figure 7 Block diagram of DWT of a signal for each level.

The Wavelet coefficients provide a compact representation of the pulmonary sounds in time and frequency. Since the pulmonary sound frequency spectrum ranges from 60 $\mathrm{Hz}$ to $2000 \mathrm{~Hz}$, the wavelet coefficients corresponding to the frequency sub-bands D1 and A6 were easily discarded and the remaining sub-bands D2-D6 were used as feature vectors for classification of the pulmonary sound signals.

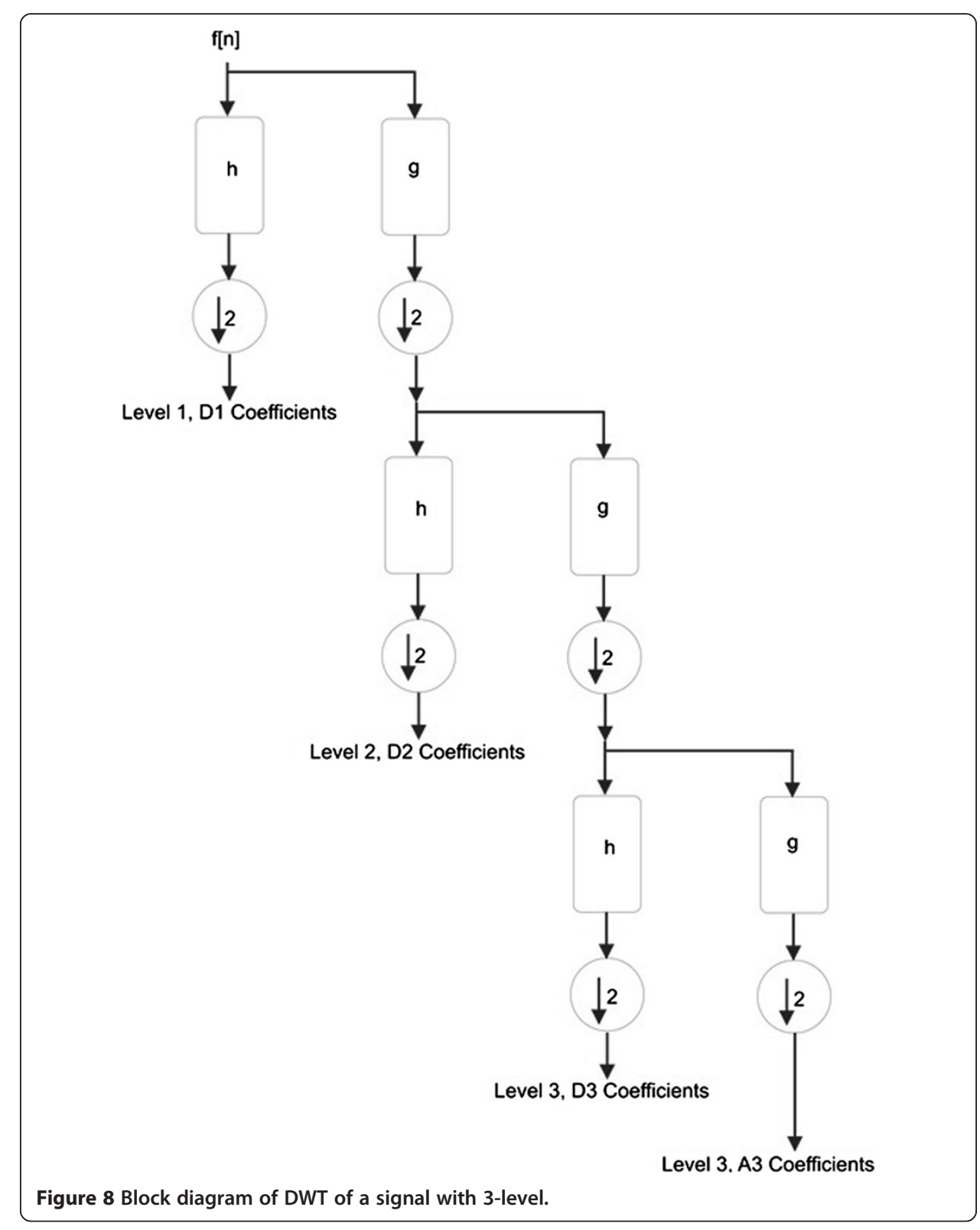




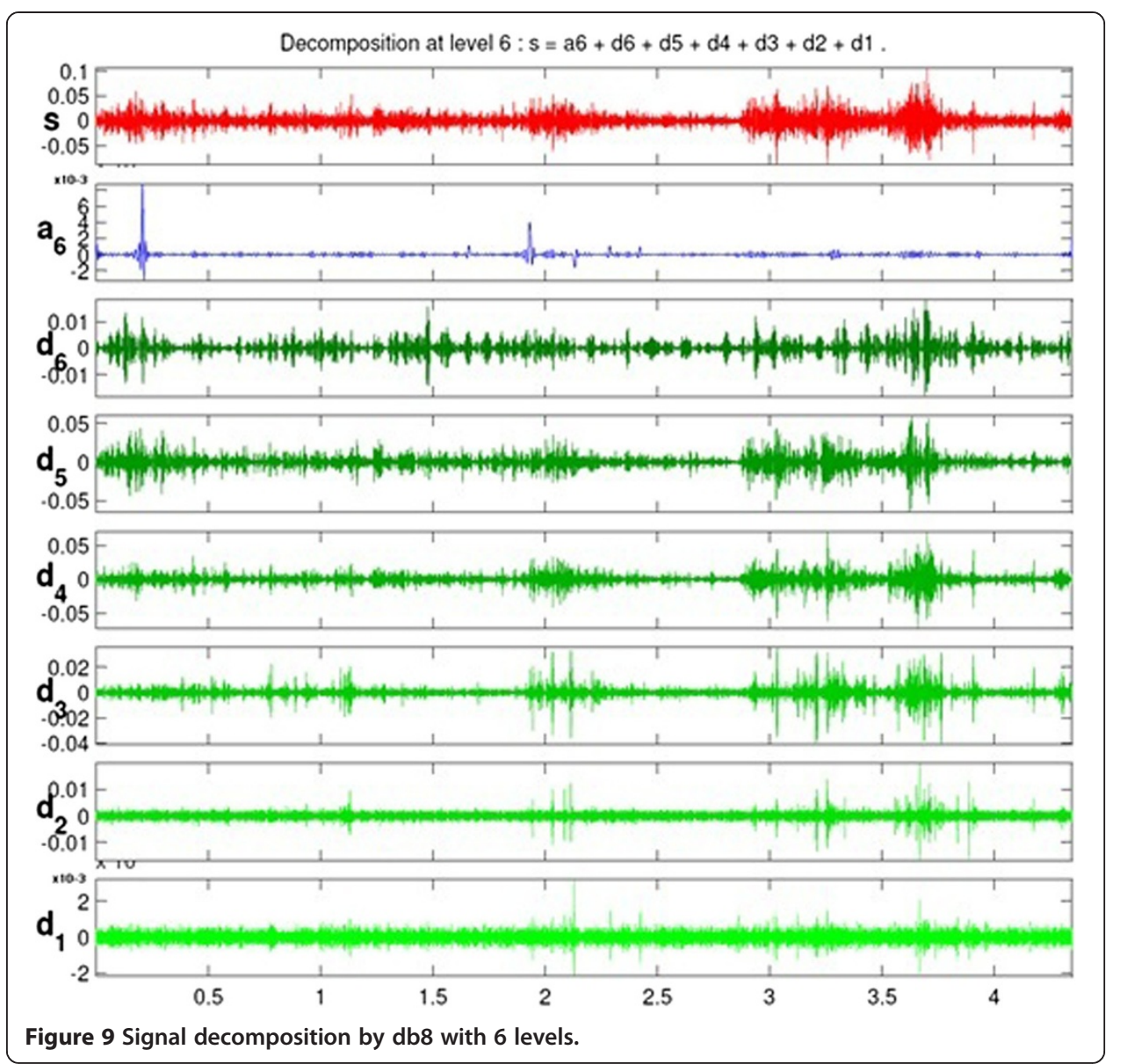

In order to further reduce the size of the feature vectors, the following statistical features were used to represent the time-frequency distribution of the pulmonary sound signals.

1. Mean values of the wavelet coefficients in each sub-band.

2. Average power of the wavelet coefficients in each sub-band.

3. Standard deviation of the wavelet coefficients in each sub-band.

4. Ratio of the absolute mean values of adjacent sub-bands.

Table 2 Frequency sub-bands in wavelet decomposition

\begin{tabular}{cc}
\hline Decomposed signal levels & Frequency range $\mathbf{( H z )}$ \\
\hline D1 & $2000-4000$ \\
D2 & $1000-2000$ \\
D3 & $500-1000$ \\
D4 & $250-500$ \\
D5 & $125-250$ \\
D6 & $62.5-125$ \\
A6 & $0-62.5$ \\
\hline
\end{tabular}


Table 3 presents the statistical features of two sample recordings: one from healthy subject and one from subject with asthma disease.

\section{Classification methods used Random forest algorithm}

Random Forest is essentially an ensemble of unpruned classification trees. It gives excellent performance on a number of practical problems, largely because it is not sensitive to noise in the data set, and it is not subject to overfitting. It works fast, and generally exhibits a substantial performance improvement over many other tree-based algorithms.

The classification trees in the Random Forest are built recursively by using the Gini node impurity criterion (Raileanu and Stoffel 2004) which is utilized to determine splits in the predictor variable. A split of a tree node is made on variable in a manner that reduces the uncertainty present in the data and hence the probability of misclassification. Ideal split of a tree node occurs when Gini value is zero. The splitting process continues until a "forest", consisting of multiple trees, is created. Classification occurs when each tree in the forest casts a unit vote for the most popular class. The Random Forest then chooses the classification having the most votes over all the trees in the forest. Pruning is not needed as each classification is produced by a final forest that consists of independently generated trees created through a random subset of the data, avoiding over fitting. The generalization error rates depend on the strength of the individual trees in the forest and the correlation between them. This error rate converges to a limit as the number of trees in the forest becomes large. Another advantage of Random Forest is that there is no need for cross validation or a separate test set to get

Table 3 Statistical feature vectors for two classes

\begin{tabular}{lccc}
\hline Levels & Features & Normal & Asthma \\
\hline D6 & Mean & -0.001752 & 0.001327 \\
& Std. Dev. & 0.016430 & 0.224913 \\
Avg. Power & Mean & 0.000272 & 0.050483 \\
& Std. Dev. & -0.000198 & -0.003998 \\
& Avg. Power & 0.029939 & 0.278092 \\
D4 & Mean & 0.000895 & 0.077270 \\
& Std. Dev. & 0.000068 & -0.001254 \\
& Avg. Power & 0.014396 & 0.186106 \\
D3 & Mean & 0.000207 & 0.034619 \\
& Std. Dev. & 0.000029 & -0.000095 \\
& Avg. Power & 0.003607 & 0.072520 \\
D2 & Mean & 0.000013 & 0.005258 \\
& Std. Dev. & 0.000009 & 0.000016 \\
& Avg. Power & 0.001441 & 0.006338 \\
& mean(abs(D6))/mean(abs(D5)) & 0.000002 & 0.000040 \\
& mean(abs(D5))/mean(abs(D4)) & 8.860058 & 0.331818 \\
& mean(abs(D4))/mean(abs(D3)) & 2.906312 & 3.187642 \\
& mean(abs(D3))/mean(abs(D2)) & 2.333392 & 13.171888 \\
& & 3.341858 & 5.805228 \\
\hline
\end{tabular}


an unbiased estimate of the classification error. Test set accuracy is estimated internally in Random Forest by running out-of-bag samples. For every tree grown in Random Forest, about one-third of the cases are out-of-bag (out of the bootstrap sample). The out-of- bag samples can serve as a test set for the tree grown on the non-out-of- bag data. The specifics of Random Forest algorithm can be summarized as follows:

1. A number $n$ is specified, which is much smaller than the total number of variables $N$ (typically $\mathrm{n} \sim \sqrt{N}$ )

2. Each tree of maximum depth is grown without pruning on a bootstrap sample of the training set

3. At each node, $n$ out of the $N$ variables are selected at random

4. The best split on these $n$ variables is determined by using Gini node impurity criterion.

Reducing $n$ reduces the strength of the individual trees in the forest and the correlation between them. Increasing it increases both. Using the out-of- bag error rate, an

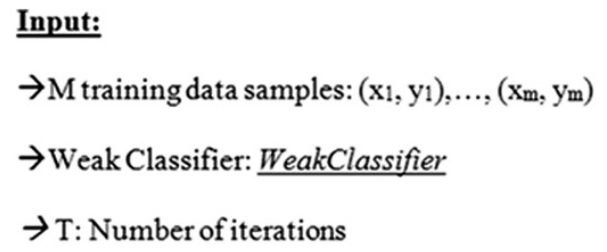

Adaboost Algorithm

Initialize $D_{1}(i)=1 / M$ for each sample if

For $\mathrm{t}=1$ to $\mathrm{T}$

Select $\mathrm{S}_{\mathrm{t}}$ from the distribution $\mathrm{D}_{\mathrm{t}}$

Train WeakClassifier with $\mathrm{S}_{\mathrm{t}}$ to obtain hypothesis $\mathrm{h}_{\mathrm{t}}$

Compute error $\varepsilon_{t}=\sum_{i: h_{t}\left(x_{i}\right) \neq y_{i}} D_{t}(\mathrm{i})$ for hypothesis $\mathrm{h}_{\mathrm{t}}$

If $\varepsilon_{t}>0.5$ then exit

Choose $\alpha_{t}=0.5 \ln \left(\left(1-\varepsilon_{t}\right) / \varepsilon_{t}\right)$

Update distribution for the $(t+1)^{\text {th }}$ iteration such that

$D_{t+1}(\mathrm{i})=D_{t}(\mathrm{i}) e^{\left(-\alpha_{t} y_{i} h_{t}\left(x_{i}\right)\right)} / Z_{t}$ where $Z_{t}=\sum_{i} D_{t}(\mathrm{i})$ is a normalization constant chosen so that $D_{t+1}$ becomes a proper distribution function.

Endfor

\section{Output:}

$H(\mathrm{x})=\operatorname{sign}\left(\sum_{t=1}^{\mathrm{T}} \alpha_{t} h_{t}(x)\right)$ is the weighted majority vote of the $\mathrm{T}$ weak hypothesis where $\alpha_{t}$ is the weight assigned to weak hypothesis $h_{t}$.

Figure 10 AdaBoost algorithm. 
optimum value of $n$ can be found. This is the only adjustable parameter to which random forests is sensitive to. The computational complexity for each tree in Random Forest is $\mathrm{O}(\mathrm{S} \log (\mathrm{S}) \sqrt{N})$, where $S$ is the number of training cases. Therefore, it can handle very large number of variables with moderate number of observations.

\section{AdaBoost Algorithm}

AdaBoost is a fast, simple and easy to use iterative algorithm which needs only one parameter to tune, i.e. number of iteration, $T$. It does not subject to over-fitting and easily identifies outliners which are either misclassified or hard to classify.

AdaBoost algorithm was first introduced by Freund and Schapire (Freund nd Schapire 1997). The algorithm takes a training set consisting of $m$ samples $\left(x_{1}, y_{1}\right), \ldots,\left(x_{m}, y_{m}\right)$ as input, where each $x_{i}$ belongs to an instance space $X$, and each label $y_{i}$ belongs to finite label space $Y=\{-1,+1\}$ for binary classification. AdaBoost generates a set of weak classifiers, and linearly combines them in an optimal way into a stronger classifier. The job of a weak classifier is to find a weak hypothesis $h_{t}: X \rightarrow\{-1,+1\}$ using samples drawn from iteratively updated distribution, $\mathrm{D}_{\mathrm{t}}$, of the training set. One of the main ideas of the AdaBoost algorithm is to maintain a weight distribution $D_{t}(i)$ on training samples $x_{i}$, (for $i=1$.. M), from which training data subsets $S_{t}$ are chosen for each consecutive hypothesis $h_{t}$. Updating distribution $\mathrm{D}_{\mathrm{t}}(\mathrm{i})$ for every iteration ensures that samples misclassified by the previous weak classifier are more likely to be included in the training data of the next weak classifier. Error of a weak classifier is measured with respect to distribution $\mathrm{D}_{\mathrm{t}}$ as $\varepsilon_{t}=\sum_{i: h_{t}\left(x_{i}\right) \neq y_{i}}$ $D_{t}(\mathrm{i})$. If it is possible for the weak classifier to use weights $\mathrm{D}_{\mathrm{t}}$ on the training dataset, then $S_{t}$ becomes $D_{t}$. Otherwise, $S_{t}$ are constructed by resampling the training data according to $\mathrm{D}_{\mathrm{t}}$. Adaboost algorithm is depicted in Figure 10. Initially, all weights of the distribution are set equally, $D_{1}(i)=1 / M$, so that all samples have equal likelihood to be selected into the first training dataset, $\mathrm{S}_{1}$. AdaBoost calls a given weak classifier algorithm iteratively in a series of rounds $\mathrm{t}=1$ to $\mathrm{t}=\mathrm{T}$. A weak hypothesis $h_{t}$ and a training error $\varepsilon_{t}$ for it are computed at each iteration, t. The distribution $D_{t}$ is next updated by $\alpha_{t}$ such that the

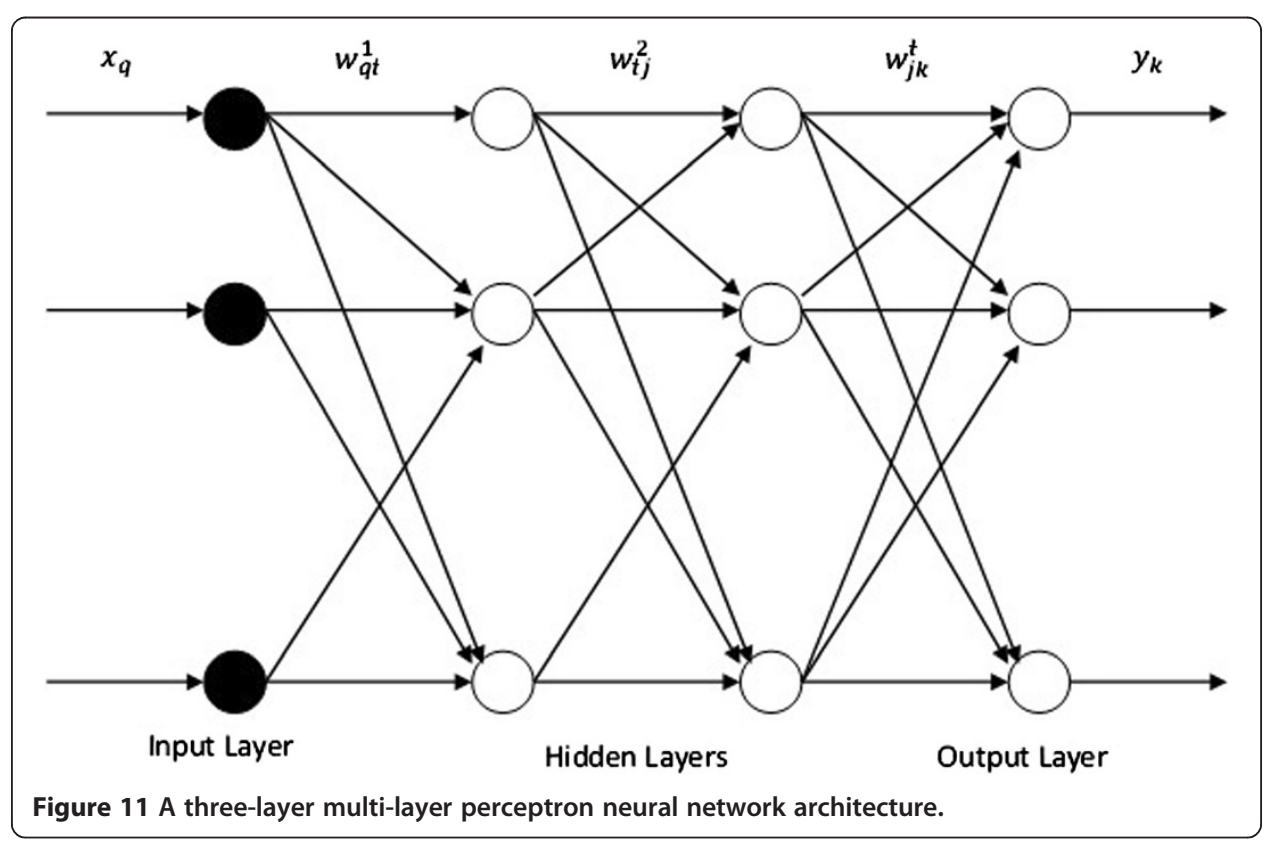


Table 4 Confusion matrix for the result of random forest classifier

\begin{tabular}{llcc}
\hline & & Prediction & \\
\hline \multirow{3}{*}{ Actual } & & Healthy & Asthma \\
& Healthy & 18 & 2 \\
& Asthma & 2 & 18 \\
\hline
\end{tabular}

weight of samples misclassified by $h_{t}$ is increased, and the weight of samples correctly classified by $h_{t}$ is decreased. Thus, AdaBoost concentrates more on difficult samples. $\alpha_{t}$ is also the weight assigned to weak hypothesis $h_{t}$ so that weak classifiers that have shown good performance during training phase have more influence at the output than others in the testing phase.

We employed Random Forest algorithm as a weak classifier in the AdaBoost algorithm to predict asthma lung sounds. Boosting Random Forest algorithm is somewhat similar to employing forests of random forests.

\section{Artificial neural networks (ANNs)}

We also included Artificial Neural Networks (ANNs) to be compatible or comparable with the pulmonary disease classification algorithms in the literature (Delen 2009 and Delen et al. 2010). Over the years, ANNs have found their way into numerous applications (Haykin 1994) ranging from pattern recognition, prediction to optimization and control systems. ANNs inspired by biological evidence are designed to solve complex problems by trying to replicate the networks of the real neurons in the human brain.

ANNs are not intelligent, but they are good at learning underlying patterns and making simple rules for complex problems without human intervention.

ANNs can be viewed as weighted directed graphs in which directed edges with weights are used to connect artificial neuron nodes. ANNs can be categorized as feedforward networks and recurrent networks. Properties of feed-forward networks are:

- They are static. i.e. for a given input, they produce only one set of output.

- They are memory-less. i.e. output is independent of the previous network state.

- They have no loops in the directed graph.

On the other hand, because of the feedback loops in the directed graph, recurrent networks are dynamic systems with memory. The most common form of feed-forward networks is multi-layer perceptron (MLPNN) which consists of multiple layers of nodes in a directed graph in which each layer is fully connected to previous and next layer. Each node in a MLPNN is a computational element that employs a differentiable nonlinear activation function such as sigmoid function to its input. Generally, a MLPNN consists of input layer, one or more hidden layers and one output layer as shown in Figure 11.

Table 5 Confusion matrix for the result of adaboosted random forest classifier

\begin{tabular}{llcc}
\hline & & Prediction & \\
\hline \multirow{3}{*}{ Actual } & & Healthy & Asthma \\
& Healthy & 18 & 2 \\
& Asthma & 2 & 18 \\
\hline
\end{tabular}


Table 6 Confusion matrix for the result of MLPNN classifier

\begin{tabular}{llcc}
\hline & & Prediction & \\
\hline \multirow{3}{*}{ Actual } & & Healthy & Asthma \\
& Healthy & 16 & 4 \\
& Asthma & 4 & 16 \\
\hline
\end{tabular}

In this paper, we used standard MLPNN in which a weight vector $w$ on the $\operatorname{arcs} A$ of network $\mathrm{N}(\mathrm{w}, \mathrm{A})$ determines a mapping $H$ from the input nodes of $\mathrm{N}$ to the output nodes of $\mathrm{N}$. Learning process in MLPNN can be viewed as iteratively updating the arc weights $\mathrm{w}$ for each input output pair in the training pattern set so that a network can efficiently perform a specific task. $w_{i j}^{l}$ in Figure 11 denotes the weight on the connection between $\mathrm{i}^{\text {th }}$ node in layer $(l-1)$ to the $\mathrm{j}^{\text {th }}$ node in layer $l . \mathrm{x}$ is the input vector and $\mathrm{y}$ is the corresponding observed output vector. During the learning process, observed output $\mathrm{y}$, generated by the network $\mathrm{N}$ may not equal to the desired output $\mathrm{d}$. Basic learning rule in MLPNN is to automatically adjust arc weights $\mathrm{w}$ such that a certain cost function is minimized. The squared-error cost function, $E=\frac{1}{2} \sum_{i=1}^{p}\left\|y^{i}-d^{i}\right\|$, which is the sum of squared differences between the observed and desired outputs for a set of $\mathrm{p}$ training patterns, $T=\left\{\left(x^{1}, d^{1}\right),\left(x^{2}, d^{2}\right),\left(x^{3}, d^{3}\right), \ldots,\left(x^{p}, d^{p}\right)\right\}$, is the most frequently used cost function to minimize total error at the output of the network. There are several learning algorithms in the literature to train a MLPNN and backpropagation (BP) algorithm (Rumelhart and McClelland 1986) is the most frequently used one. The BP algorithm is a gradient-descent method which searches an error surface for points with minimum error. Optimization methods such as Levenberg-Marquardt (Levenberg 1944 and Marquardt 1963) and quasi-Newton (Broyden 1969) have also been used in recent years. In all these algorithms, after introducing a new input-output pair to the network from the training dataset, each weight $w_{i j}^{l}$ on arcs of the network is modified by adding an increment $\Delta w_{i j}^{l}$. How $\Delta w_{i j}^{l}$ is computed depends on the training algorithm. For our prediction system, we chose Levenberg-Marquardt training algorithm due its fast convergence property.

\section{Results and discussion}

In this study, we used equal number of healthy subjects and asthma patients who had been diagnosed according to GINA guidelines with symptoms compatible with asthma and current or previous demonstration of airway hyper-responsiveness (GINA 2010). During the data collection phase of the study, the subjects were in relaxed position with the microphones attached to right and left posterior bases of their lungs.

During the analysis phase, both inspiration and expiration cycles were considered separately for each subject. However, in the classification part, we used only expiration

Table 7 Values of statistical performance parameters for the random forest and adaboosted random forest classifier

\begin{tabular}{cc}
\hline Statistical performance parameters & Values (\%) \\
\hline Specificity & 90 \\
Sensitivity & 90 \\
Accuracy & 90 \\
\hline
\end{tabular}


Table 8 Values of statistical performance parameters for the MLPNN classifier

\begin{tabular}{cc}
\hline Statistical performance parameters & Values (\%) \\
\hline Specificity & 80 \\
Sensitivity & 80 \\
Accuracy & 80 \\
\hline
\end{tabular}

cycles over right and left posterior bases of the lungs, which provided more specific information about the pulmonary diseases and helped us to obtain better classification results.

Since our pulmonary signal dataset was small, in order to avoid overfitting, we applied leave one out (LOO) cross validation procedure by splitting the whole dataset as training and test set. Each test set is constructed by taking one sample from the original dataset; the remaining samples are used to learn the model. This process is repeated for each sample in the dataset. Thus, for $n$ samples in the dataset, $n$ different training set and $n$ different test set are created.

Classification results of the all algorithms were presented using confusion matrixes. A confusion matrix shows the number of correct and incorrect predictions made by the classifier compared with the actual classifications given in the original data set. The confusion matrix is a two-dimensional $n$-by- $n$ matrix, where $n$ is the number of classes in the output variable. In this study, number of classes was only two: healthy and asthma. Each column of the matrix represents the predictions, while each row represents the actual classifications. By using confusion matrix, one can see whether the prediction model is mislabeling classes, and whether the prediction of classed is based towards one class in expense of other.

The confusion matrices showing the results of the Random Forest, AdaBoosted Random Forest and MLPNN classification algorithms are given in Table 4, Table 5 and Table 6, respectively. According to the confusion matrix, two normal sound recordings were classified incorrectly by the random forest and AdaBoosted random forest algorithms as asthma disease (i.e., false positive), and two recordings having asthma disease were classified as normal recordings (i.e., false negative).

The performance of a classifier was determined using the computation of the following statistical parameters:

Specificity: Number of correctly classified recordings belonging to healthy subjects/ Total number of recordings belonging to healthy subjects,

Sensitivity: Number of correctly classified recordings belonging to subjects with asthma disease/Total number of recordings belonging to subjects with asthma disease,

Accuracy: Number of correctly classified recordings/Total number of recordings.

The values of these statistical performance parameters for each classification algorithm are shown in Tables 7 and 8. The total classification accuracy of the Random Forest and

Table 9 Confusion matrix for the result of random forest and adaboosted random forest classifiers with FEV and FVC1 values added to the feature vector

\begin{tabular}{llcc}
\hline & & Prediction & \\
\hline \multirow{3}{*}{ Actual } & Healthy & Healthy & Asthma \\
& Asthma & 19 & 2 \\
& & 1 & 18 \\
\hline
\end{tabular}


Table 10 Values of statistical performance parameters for the random forest and adaboosted random forest classifiers with FEV and FVC1 values added to the feature vector

\begin{tabular}{cc}
\hline Statistical performance parameters & Values (\%) \\
\hline Specificity & 95.0 \\
Sensitivity & 90.0 \\
Accuracy & 92.5 \\
\hline
\end{tabular}

AdaBoosted Random Forest classifiers were $90 \%$. The number of variables to split on at each node, $n$, was found to be 5 . This value gave the smallest OOB error rate. For the AdaBoosted Random Forest algorithm, maximum iteration, T, was chosen as 30. For the MLPNN algorithm, we created a network with one hidden layer which consists of 15 nodes. During the training process, learning rate and momentum was chosen as 0.3 and 0.2 , respectively.

We also extended the feature vector by adding FVC and FEV1 clinical values for each patient. Tables 9 and 10 show the results of classification and performance of the parameters for Random Forest and AdaBoosted Random Forest algorithms. As can be seen in Table 1, there are two cases where FEV1 and FVC values are missing. Random Forest was also used to estimate these missing values by first assigning them to the median of all values in the same class. Later, a full random forest tree was generated and missing values were re-estimated by using the proximities between the cases which the missing values belong to and non-missing value cases. This process was repeated until the missing values converge.

The results of classification and performance of the parameters for MLPNN algorithm is shown in Table 11 and Table 12, respectively.

One important feature of the Random Forest algorithm is to compute importance of attributes in the feature vector. It is particularly interesting to identify the attributes that contribute the most to classify asthma patients. One way to determine the most effective attributes by using Random Forest algorithm is to score attributes based on the levels of nodes that use them to split data. Attributes used in higher levels in the tree contributes more than those used in lower levels.

Out of the 21 attributes that we used in this study, including FEV1 and FVC, the top five important attributes are shown in Table 13.

\section{Conclusion}

In this study, we developed predictive models using three machine learning algorithms to diagnose an asthma patient based solely on the sounds acquired from the chest of the patient in a clinical laboratory. We employed a four-stage process to analyzed pulmonary sound signals: normalization, wavelet decomposition, feature extraction, and

Table 11 Confusion matrix for the result of MLPNN classifier with FEV and FVC1 values added to the feature vector

\begin{tabular}{llcc}
\hline & & Prediction & \\
\hline \multirow{3}{*}{ Actual } & & Healthy & Asthma \\
& Healthy & 17 & 3 \\
& Asthma & 3 & 17 \\
\hline
\end{tabular}


Table 12 Values of statistical performance parameters for the MLPNN classifier with FEV and FVC1 values added to the feature vector

\begin{tabular}{cc}
\hline Statistical performance parameters & Values (\%) \\
\hline Specificity & 85.0 \\
Sensitivity & 85.0 \\
Accuracy & 85.0 \\
\hline
\end{tabular}

the classification. Although, the performances varied slightly, ensemble models (i.e., Random Forest and AdaBoost combined with Random Forest) achieved better prediction results (about 90\%) than the artificial neural networks models (with about $80 \%$ predictive accuracy).

Our results show that non-invasive, computerized lung sound analysis that rely on low-cost microphones and an embedded real-time microprocessor system could help physicians to make faster and better diagnostic decisions, especially in situations where $\mathrm{x}$-ray and CT-scans are not reachable or not available. The purpose of these types of computerized system (also commonly referred to as evidence based medicine systems) is not to replace, but to augment the diagnostic capabilities of physicians.

There are several limitations of our study. First, the number of subjects was small. Even though, collection of these types of data in a clinical environment is challenging and time consuming (hence the smaller number of subjects used in many of the previous studies), it is always better to have as large of a datasets as possible to better generalize the findings of machine learning techniques. Second, subjects in the clinical laboratory allowed having spontaneous respiration; that is, their respiration speed and volume was not externally managed or controlled. Third, in this study we focused on only one pulmonary disease, asthma, instead of trying to diagnose all common pulmonary diseases. Finally, we choose to used only three popular machine learning techniques. Our future research directions will be focusing on mitigating these limitations. Specifically, we are going to acquire more data, and try to diagnose other common pulmonary diseases such as chronic obstructive pulmonary disease, pneumonia, idiopathic pulmonary fibrosis.

The results of our study suggest that asthma disease can be classified with an accuracy of approximately $90 \%$ by combining discrete wavelet transformation with different machine learning methods. There was not any performance difference between AdaBoosted Random Forest and Random Forest classifiers. However, when compared to MLPNN, both of them perform better. Slight performance improvements were observed for all classification algorithms after FEV and FVC1 clinical values were added to the feature vector.

The practical implications of this study are not limited to diagnosing problems in the field of healthcare/medicine. There are many problems where data and models (i.e.,

Table 13 Importance of attributes

\begin{tabular}{lc}
\hline Attribute name & Raw score \\
\hline Mean(abs(D6))/Mean(abs(D5)) & 6.08 \\
FEV1 & 3.74 \\
FVC & 3.62 \\
Mean(abs(D5))/Mean(abs(D4)) & 2.26 \\
Average Power of (D5) & 1.76 \\
\hline
\end{tabular}


analytics) can be used to either fully automate or semi-automate decision situations. Such data/evidence-based practices would greatly increase the accuracy and timeliness of decisions while freeing up critical human resources (i.e., knowledge workers). This in turn would increase the viability, competitiveness and sustainability of today's organizations that are required to do more and better with increasingly more stringent resource constraints.

\section{Competing interests}

The authors declare that they have no competing interests.

\section{Authors' contributions}

All four authors contributed to the underlying research as well as the authoring of the manuscript roughly equally. All authors read and approved the final manuscript.

\section{Acknowledgments}

This study is supported by Scientific and Technological Research Council of Turkey (TUBITAK) under the project number 104M308.

\section{Author details}

${ }^{1}$ Department of Computer Engineering, Faculty of Engineering, Fatih University, Istanbul, Turkey. ${ }^{2}$ Department of Genetics and Bioengineering, Faculty of Engineering, Fatih University, Istanbul, Turkey. ${ }^{3}$ Department of Pulmonary Diseases, Faculty of Medicine, Gaziantep University, Gaziantep, Turkey. ${ }^{4}$ Department of Management Science and Information Systems, Spears School of Business, Oklahoma State University, Stillwater, OK, USA.

Received: 12 August 2013 Accepted: 5 September 2013

Published: 19 February 2014

\section{References}

Ali, J, Summer, WR, \& Levitzky, MG. (2009). Pulmonary pathophysiology (3rd ed.). New York: McGraw-Hill Medical. Breiman, L. (2001). Random Forests. Machine Learning, 45, 5-32.

Broyden, CG. (1969). A new double-rank minimization algorithm. Notices of the American Mathematical Society, 16, 670-684. Çatmakaș, Z, Köse, IH, Toker, O, \& Öz, HR. (2009). Towards an ARM based low cost and mobile biomedical device test bed for improved multi-channel pulmonary diagnosis. In 4 th European Conference of the International Federation for Medical and Biological Engineering (pp. 1108-1112). Berlin Heidelberg: Springer.

Daubechies, I. (1990). The wavelet transform, time-frequency localization and signal analysis. IEEE Transactions on Information Theory, 36, 961-1005.

Delen, D. (2009). Analysis of cancer data: a data mining approach. Expert Systems, 26(1), 100-112.

Delen, D, Fuller, C, McCann, C, \& Ray, D. (2009). Analysis of healthcare coverage: a data mining approach. Expert Systems with Applications, 36(2), 995-1003.

Delen, D, Oztekin, A, \& Kong, Z. (2010). A machine learning-based approach to prognostic analysis of thoracic transplantations. Artificial Intelligence in Medicine, 49(1), 33-42.

Doyle, M. (1994). Analysis of lung sounds using neural networks. Master of Science Thesis: Vanderbilt University.

Earis, JE, \& Cheetham, BMG. (2000). Current methods used for computerized respiratory sound analysis. European Respiratory Review, 10, 586-590

Freund, Y, \& Schapire, RE. (1997). A decision-theoretic generalization of on-line learning and an application to boosting. Journal of Computer and System Sciences, 55(1), 119-139.

Furber, S. (2000). ARM System-on-chip architecture. Parlow: Addison-Wesley Professional.

Gavriely, N. (1995). Breath sounds methodology. Boca Raton: CRC Press.

GINA. (2012). Global initiative for asthma, GINA report, global strategy for asthma management and prevention. www. ginasthma.org/local/uploads/files/GINA_Report_March13.pdf.

Haykin, S. (1994). Neural networks: a comprehensive foundation. New York: MacMillan Collage Publishing.

Jensen, A, \& Harbo, AC. (2001). Ripples in mathematics, the discrete wavelet transform (1st ed.). Berlin: Springer Verlag.

Kahya, YP, Yeginer, M, \& Bilgic, B. (2006). Classifiying respiratory sounds with different feature set. Conf Proc IEEE Eng Med Bio Soc, 1, 2856-2859.

Kandaswamy, A, Kumar, RC, Sathish, C, Jayaraman, S, \& Malmurugan, N. (2004). Neural classification of lung sounds using wavelet coefficients. Computers in Biology and Medicine, 34, 523-537.

Kester, W. (2005). Data conversion handbook. Burlington: Newnes.

Levenberg, K. (1944). A method for the solution of certain problems in least squares. Quart Appl Math, 2, 164-168.

Mallat, S. (2009). A wavelet tour of signal processing (3rd ed.). Boston: Academic.

Marquardt, D. (1963). An algorithm for least squares estimation of non-linear parameters. SIAM Journal on Applied Mathematics, 11, 431-441.

Martinez, AM, \& Kak, AC. (2001). PCA versus LDA. IEEE Transactions on Pattern Analysis and Machine Intelligence, 23, 228-233.

Masoli, M, Fabian, D, Holt, S, \& Beasley, R. (2010). Global burden of asthma, medical research institute of New Zeland. New Zeland: Wellington.

Mohammed, B. (2009). Pattern recognition methods applied to respiratory sounds classification into normal and wheeze classes. Computers in Biology and Medicine, 39, 824-843.

Murphy, RL, Vyshedskiy, A, Power, VA, Bana, D, \& Marinelli, A. (2004). Wong. Automated lung sound analysis in patients with pneumonia, Resp. Care, 49(12), 1490-1497. 
Ono, H, Tabiguchi, Y, Shinoda, K, Sakamoto, TS, \& Kudoh, A. (2009). Gemma: Evaluation of the usefulness of spectral analysis of inspiratory lung sounds recorded with phonopneumography in patients with interstitial pneumonia. Journal of Nippon Medical School, 76, 67-75.

Oz, HR, Kara, N, \& Bayram, N. (2009). Analysis of pulmonary diseases using genetic programming. Journal of Applied Biological Sciences, 3, 28-31.

Oz, HR, Kara, N, \& Bayram, N. (2010). Comparison of different clustering methods for pulmonary sounds. J Comp Biomed Eng, 1, 9-14.

Oztekin, A, Delen, D, \& Kong, Z. (2009). Predicting the graft survival for heart-lung transplantation patients: an integrated data mining methodology. International Journal of Medical Informatics, 78(12), e84-e96.

Pesu, L, Helisto, P, Ademovic, E, Pesquet, JC, Saarinen, A, \& Sovijarvi, ARA. (1998). Classification of respiratory sounds based on wavelet packet decomposition and learning vector quantization. Technology and Health Care, 6, 65-74.

Raileanu, L, \& Stoffel, K. (2004). Theoretical comparison between the gini index and information gain criteria. Annals of Mathematics and Artificial Intelligence, 41, 77-93.

Rumelhart, DE, \& McClelland, JL. (1986). Parallel distributed processing: exploration in the microstructure of cognition. Cambridge, MA: MIT Press.

Sankur, B, Kahya, YP, Guler, CE, \& Engin, T. (1994). Comparison of AR-based algorithms for respiratory sounds classification. Computers in Biology and Medicine, 24, 67-76.

doi:10.1186/2193-8636-1-6

Cite this article as: Emanet et al: A comparative analysis of machine learning methods for classification type decision problems in healthcare. Decision Analytics 2014 1:6.

Submit your manuscript to a SpringerOpen ${ }^{\circ}$ journal and benefit from:

- Convenient online submission

- Rigorous peer review

- Immediate publication on acceptance

- Open access: articles freely available online

- High visibility within the field

- Retaining the copyright to your article

Submit your next manuscript at $\gg$ springeropen.com 